\title{
Frontiers in epigenetics medicine: foreword
}

\author{
Miniseries assembled from a symposium organized by the Study Group on Epigenetics \\ Medicine held at the 77th Annual Meeting of the Japanese Society for Hygiene, 2007
}

\author{
Yasuhito Yuasa
}

Published online: 15 December 2007

(C) The Japanese Society for Hygiene 2008

Epigenetics refers to heritable changes in gene expression that occur without an alteration in DNA sequence. Such changes include the methylation of genomic DNA and modifications of chromosomal histone proteins, such as acetylation and methylation. DNA methylation occurs at the carbon-5 position of symmetrical $\mathrm{CpG}$ dinucleotides. The state of DNA methylation is maintained after cell division through the activity of DNA methyltransferase 1, which methylates hemimethylated $\mathrm{CpG}$ in the daughter cells. Hypermethylation in gene promoters causes heritable silencing and, consequently, the inactivation of genes. Histone modifications, on the other hand, involve the addition of acetyl, methyl or other groups to protruding histone tails. These modifications constitute the potential "histone code" that underlies a specific chromatin structure, which in turn affects the expression of adjacent genes. The epigenetic status shows plasticity both during development and postnatally, depending on environmental and other factors. Thus, changes in epigenetic status could contribute not only to developmental human disorders, but also to postnatal and even adult diseases.

In the first article of this miniseries, Dr. Takeo Kubota, Department of Epigenetics Medicine, University of Yamanashi, introduces congenital diseases with disorders in well-established epigenetic mechanisms and discusses pervasive developmental disorders that may be caused by epigenetic mechanisms. Cancers arise as a consequence of multiple genetic and epigenetic alterations. The group of Dr. Toshikazu Ushijima, Carcinogenesis Division, National Cancer Center Research Institute, demonstrated the concept of an "epigenetic field for cancerization" for gastric cancers in a large-scale analysis of gastric mucosae of healthy individuals and gastric cancer patients. Next, Dr. Tadafumi Kato and his colleague, the Laboratory for Molecular Dynamics of Mental Disorders, RIKEN Brain Science Institute, reported epigenetics in mood disorders by discussing several sets of data, including clinical studies relevant to depression, the role of histone modification in antidepressive treatment action, the role of DNA methylation in mood regulation, and DNA methylation analysis in the postmortem brain. In the final article, Dr. Hiroyuki Mano, Division of Functional Genomics, Jichi Medical University, suggested that epigenetic changes play an important role in the development of cardiac hypertrophy and heart failure based on a study involving the coupling of chromatin immunoprecipitation to high-throughput profiling systems of cardiac myocytes in normal as well as affected hearts.

Thus, epigenetic changes in human diseases have started to make an impact on their prevention, diagnostics, and therapeutics. Given the developments in the field of epigenetics, researchers in environmental health and preventive medicine will have to pay attention to Epigenetics Medicine.

\footnotetext{
Y. Yuasa ( $\square)$

Chairperson of the Study Group on Epigenetics Medicine,

Department of Molecular Oncology,

Graduate School of Medicine and Dentistry,

Tokyo Medical and Dental University, Tokyo, Japan

e-mail: yuasa.monc@tmd.ac.jp
} 